\title{
Review of: "Effects of blood urea nitrogen independent of the estimated glomerular filtration rate on the development of anemia in non-dialysis chronic kidney disease: The results of the KNOW-CKD study"
}

Yukio Maruyama

Potential competing interests: The author(s) declared that no potential competing interests exist.

The authors investigated the association between BUN and prevalence or incidence of anemia 2,196 CKD 1-5 patients. Although the results were meaningful, there were several problems. Especially, they should clarify the purpose of the study. Erythropoietin (EPO) is produced by renal erythropoietin-producing (REP) cells in renal interstitium, therefore the EPO production is not directly associated with GFR. Why did they investigate the association between BUN and anemia? How do they reflect their results in the management of renal anemia? Do the authors recommend restriction of protein intake for the prevention of anemia? It is well-known that BUN is not a specific marker of renal insufficiency, and could enhance not only protein intake but exercise, surgical stress, consumptive disease or corticosteroid therapy. However, they treated only protein intake as a confounding factor in multivariate analysis.

The influence of two main treatment for renal anemia, ESA administration and iron supplementation, is concerned, because the discretion of the use of these drugs are usually left to the physicians. They took account of the use of ESA or iron supplement in multivariate analysis. However, adjustment for the dose of these drugs is desirable. 